\title{
Corticosteroid Injections - A Viable Treatment for Multiple Trigger Fingers: A Case Report
}

\author{
Dotun Musiliu Kadir ${ }^{1 *}$, Bola Abdulkadir Ahmed ${ }^{2}$ and Eniola Risikat Kadir ${ }^{3}$ \\ ${ }^{1}$ Department of Surgery, Orthopedic Unit, General Hospital, Ilorin, Kwara State, Nigeria \\ ${ }^{2}$ Department of Orthopedics, University of Ilorin Teaching Hospital, Ilorin, Kwara State, Nigeria
}

${ }^{3}$ Department of Human Anatomy, University of Ilorin, Ilorin, Kwara State, Nigeria

*Corresponding author: Dotun Musiliu Kadir, Department of Surgery, Orthopedic unit, General Hospital, Ilorin, Kwara State, Nigeria, Tel: +2348023110989; E-mail: dotunk@yahoo.com

Received Date: Mar 24, 2018; Accepted Date: Apr 02, 2018; Published Date: Apr 10, 2018

Copyright: $\odot 2018$ Kadir DM, et al. This is an open-access article distributed under the terms of the Creative Commons Attribution License, which permits unrestricted use, distribution, and reproduction in any medium, provided the original author and source are credited.

\section{Abstract}

A case of multiple trigger fingers is presented in a 56 y old diabetic woman who refused operative management which is the main stay of treatment. Both hands were involved; three on the left and two on the left. The thumb was spared. She was therefore managed conservatively with corticosteroid infiltration at the A1 pulley which had to be repeated but with long term satisfactory result.

Keywords: Multiple trigger finger; Conservative management; Corticosteroid injections

\section{Introduction}

Trigger finger (TF) is a stenosing tendosynovitis in which the flexor tendon sheath thickens apparently spontaneously entrapping the tendon and locks the finger in a flexed position at the retinacula of the flexor tendons of the fingers and thumb in the palm [1]

The flexor muscles are strong enough to push the tendon through the stenosed A1 pulley at the level of the metacarpophalangeal joint but the extensor muscles may not be powerful enough to extend them [2]. The finger now snaps as it passes through the constriction and locks in a position of flexion at the proximal interphalangeal joint from which attempts to passively extend the fingers are painful [3].

The underlying cause is unknown but suggested etiological factors include anatomical deviations, occupational, multi-factorial and genetic factors. It's known to be more common in patients with diabetes [4,5]. Other common associated conditions include rheumatoid arthritis, renal disease, gout, carpal tunnel and hyperthyroidism. It is commonly seen in middle aged women, diabetics and frequently involves the middle and ring finger but any finger may be involved [5-7]. There are two types; primary, the commonest is usually idiopathic and the secondary which is seen in patients with chronic diseases $[3,6]$. The life time risk of developing trigger finger is between $2-3 \%$ [8].

It is commoner in the dominant hand and any digit may be involved [9]. The most frequent is in this order - the ring, middle fingers and thumb. Sometimes multiple fingers are affected in the same hand or both hands.

The condition is managed either operatively or conservatively. However, conservative management is usually the first line of management, but when this fails, surgical options in form of soft tissue release is done. Conservative treatment includes activity modification, splinting, physiotherapy, non-steroidal anti-inflammatory drugs
(NSAIDs) and corticosteroid injections (the mainstay of non-operative treatment) $[3,10]$. We present a case of multiple trigger fingers of both hands in a 56 year old woman which was managed non-operatively.

\section{Case Report}

$\mathrm{AO}$ is a 56 year old female trader who presented to the surgical outpatient clinic with a 4 month history of pain and discomfort in both hands and difficulty in extending the fingers after they are have been clenched in a fist. She noticed these symptoms in the index, ring and little finger on the right hand and on the ring and little finger on the left. The ring finger was the first finger affected and later the middle and then the little finger (Figure 1). Pain was noticed later when it became more difficult extending the fingers. This has made activities of daily living stressful for her. She is a trader who works for 8-9 hours daily and the worsening symptoms have hindered her work over time. No history suggestive of repetitive movements of the hand or use of vibrating tools or suggestive of carpal tunnel. Past medical history revealed she has been having tingling sensations in the hands and fingers with cervical radiographs suggestive of cervical spondylosis. She is a known diabetic who is not regular on medication and attends diabetic clinic once in a while. She is post-menopausal. Not a known hypertensive or asthmatic.

On examination, she is a middle aged woman, not in painful distress, not pale, afebrile, not dehydrated. Vital signs were normal. The hands show no remarkable abnormalities on extension. On making a fist, she was unable to extend the middle, ring and little finger on the right hand and similarly on the ring and little finger on the left hand. All affected fingers show the classical triggering on attempts to passively extend them. There was some mild associated pain while attempting to extend the finger and thickening/nodules were palpated on the MCP joint of both ring fingers only. Both thumbs were normal.

An impression of multiple trigger fingers was made and attempts to rule out rheumatoid arthritis as a cause were negative. Fasting blood sugar was elevated at $11.2 \mathrm{mmol} / \mathrm{l}$ while a test for rheumatoid factor was negative. She was offered open surgical release at the A1 pulleys of 
the affected digits as the preferred treatment in view of the diabetic comorbidity and multiple digit involvement. She however refused and was then offered corticosteroid injections.

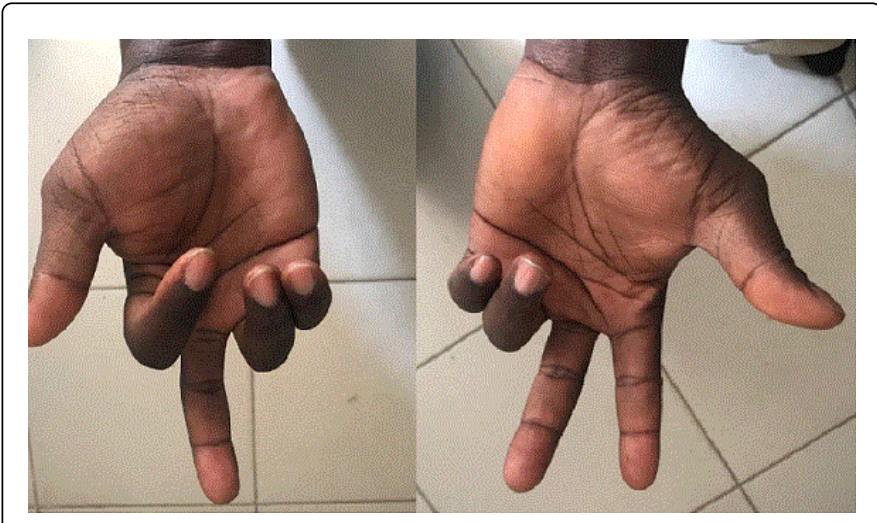

Figure 1: Clinical photograph of trigger finger (3 on the right hand and 2 on the left hand) taken separately as a result of elicited pain.

Steroid infiltration was carried out with $0.8-1 \mathrm{ml}$ of a mixture of 40 $\mathrm{mg}$ Triamcinolone $(1 \mathrm{ml})$ in $1 \mathrm{ml}$ plain $2 \%$ Lidocain. Infiltration was done under sterile conditions by inserting the needle perpendicularly over the thickened pulley and into $\mathrm{d}$ tendon. Once inside the tendon, the needle is gradually withdrawn until it's within the tendon sheath and the mixture is then injected. Same procedure was carried out for the other fingers. A light bandage is applied for comfort and oral analgesics prescribed.

She was seen in the follow up clinic a week later and all symptoms had resolved as shown by the ability to extend all fingers normally without pain or triggering.

She presented 8 months later with a month's history of recurrence in all 5 fingers as before. Surgical release was offered but she insisted on steroid infiltration which she had. This was followed with several sessions of physiotherapy. It is now 14 months after the second injection and patient has been symptom free.

\section{Discussion}

The A1 pulley is a joint (annular) pulley which originates from the volar plate of the metacarpophalangeal joint and lies in the flexor groove in the deep transverse metacarpal ligament [11]. It is one of 5 pulley systems in the hand which form a fibro-osseous canal on the palmer aspect of the hand through which the flexor tendons pass through and as such function is to guide the flexor tendons during muscle contraction and thus prevent bowstringing [11]. TFs develop as a result of minimal inflammatory changes such as chrondrocitic proliferation, increased matrix gylcosaminoglycans within the synovial sheath and hypertrophy of the A1 pulley as a result of the fibrocartiligenous metaplasia thus creating a discrepancy between the tendon and its sheath [9].

The condition is most common in women $(\mathrm{M}: \mathrm{F}=1: 2.5)$ during the fifth decade of life as reported by several studies and can occur in any age group $[3,5,10]$. Ratios of up to six times in women have been reported [3]. It is known that young age is a poor prognostic factor and this group of individuals also present with multiple digit involvement [10]. Our patient falls within the age group where TF is most common. Marks et al., Robert et al and Cornelius et al found that the most common digit involved is the thumb followed by the middle and ring fingers with subtle differences in the index and little fingers $[5,6,10]$. This distribution is usually in patients with single digit involvement. This does not conform in those with multiple involvement [3]. Multiple finger involvement has an incidence of between $20-24 \%$ and is usually associated with co-morbidity [6]. In our patient, there was multiple digit involvement limited to the index, ring and little fingers on the right hand and the ring and little fingers on the left. Bilateral involvement causes severe discomfort with regards to activities of daily living or profession [7]. And this was the case in our patient who only presented when her daily activities were hindered. Masoud et al reported a case of a musician with six trigger fingers involving five fingers on the left and one on the right which hindered his craft [7].

It has been a long held concept that occupation has a role to play in the development of TF however this claim has not been substantiated. Repetitive hand movements and occupations involved with vibrating tools are key suspects [3,9]. However Trezius et al found no correlation between TF and occupation and suggested that the majority of TF develop from reasons unrelated to work [9]. Our patient is a trader who does not use vibrating tools neither does she perform activities that require repetitive hand movements.

Amongst the risk factors in the development of TF is the presence of co-morbidities most especially diabetes mellitus as in the index case. Others include carpal tunnel, rheumatoid disease, hypothyroidism, renal disease, gout etc. $[9,12]$. Collagen diseases have also been associated with the development of multiple TFs [3]. These associated co-morbid medical conditions are more commonly seen in women [6]. It is not completely understood why TFs develop in diabetics but it is assumed that they have poor microvascular tissue bed and hence an abnormal cellular response to the corticosteroid [13]. It was also found that diabetics are more likely to develop multiple TFs, more likely to require surgery, are difficult to treat and treatment is more likely to fail [13]. In our patient, many of these factors were present.

The patient preferred corticosteroid injections over surgical release at the A1 pulley in spite of the documented success rates recorded from the latter $[7,8]$. Success rates of surgical release are between $90-100 \%$ [3]. However administration of corticosteroid which is the main stay of conservative management has also recorded rates which range from $57-97 \%[3,6,14]$. It has also been documented that the success rates of corticosteroid injection increase with the number of injections $[5,10,13]$. Single corticosteroid injections have produced success rates of $34-42 \%$ [8]. This success is better in single (76\%) rather than in multiple $(52 \%)$ trigger fingers $[10,13]$. Failure of conservative management is commoner in the male sex, multiple digit involvement, patients with chronic diseases and in young people; this failure usually occurs in the first 2 years [10]. Many authors recommend surgical release of the A1 pulley after 2-3 corticosteroid injections might have failed and rarely 4 or 5 doses in peculiar circumstances [5,8]. It is also advocated that multiple digital involvement be managed operatively due to the high failure rates of conservative management [13]. Our patient was pain and trigger free for eight months before a recurrence which necessitated a second injection, and she has been symptom free for 14 months thereafter.

\section{Conclusion}

Corticosteroid injection of trigger finger is a viable treatment option in the non-operative management of multiple digit involvement and in patients who are unfit or refuse operative management. 
Citation: Kadir DM, Ahmed BA and Kadir ER (2018) Corticosteroid Injections - A Viable Treatment for Multiple Trigger Fingers: A Case Report. Med Rep Case Stud 3: 154. doi:10.4172/2572-5130.1000154

Page 3 of 3

\section{References}

1. Ebenezer J (2010) Chapter 30: Regional conditions of the upper limb. Textbook of Orthopedics (4thedn), Jaypee, pp: 390-391.

2. Warwick D, Dun R (2010) Chapter 16: The Hand. In Appley's System of Orthopeddics (9thedn), Holder Arnold, pp: 323-324.

3. Makkouk AH, Oetgen ME, Swigart CR, Dodds SD (2008) Trigger finger: etiology, evaluation, and treatment. Curr Rev Musculoskelet Med 1: 92-96.

4. Lee YK, Kam BS, Lee KW, Kim WJ, Choy WS (2007) Ten Trigger Fingers in an Adult Man: A Case Report. J Korean Med Sci 22: 170-172.

5. Schubert C, Hui-Chou HG, See AP, Deune EG (2013) Corticosteroid injection therapy for trigger finger or thumb: a retrospective review of 577 digits. HAND 8: 439-444.

6. Marks MR, Gunther SF (1989) Efficacy of cortisone injection in treatment of trigger fingers and thumbs. J Hand Surg Am 14: 722-727.

7. Yavari M, Hassanpour SE, Mosavizadeh SM (2010) Multiple Trigger Fingers in a Musician: A Case Report. Archives of Iranian Medicine 13: 251-252.
8. Nesbitt P, Jamil W, Jesudason P, Muir L (2013) A case of Multiple Bilateral Trigger Digits: Injection or Surgery? World Journal of Medical and Surgical Case Reports 2: 92013

9. Trezies AJ, Lyons AR, Fielding K, Davis TR (1998) Is occupation an aetiological factor in the development of trigger finger? J Hand Surg $\mathrm{Br}$ 23: 539-540.

10. Wojahn RD, Foeger NC, Gelberman RH, Calfee RP (2014) Long-Term Outcomes Following a Single Corticosteroid Injection for Trigger Finger. J Bone Joint Surg Am 96: 1849-1854.

11. https://en.wikipedia.org/wiki/Annular_ligaments_of_fingers.

12. Stahl S, Kanter Y, Karnielli E (1997) Outcome of Trigger Finger Treatment in Diabetes. J Diab Comp 21: 287-290.

13. Dala-Ali BM, Nakhdjevani A (2012) The Efficacy of Steroid Injection in the Treatment of Trigger Finger. Clin Orthop Surg 4: 263-238.

14. Brito JL, Rozental TD (2010) Corticosteroid Injection for Idiopathic Trigger Finger. J Hand Surg 35: 831-833. 